\title{
EDITORIAL
}

\section{Letter from America: how will health reform affect psychiatric care?}

\author{
Steven S. Sharfstein
}

Steven S. Sharfstein is President and Chief Executive Officer of Sheppard Pratt Health System in Baltimore, Maryland, and Vice Chair, Department of Psychiatry, University of Maryland, USA. Correspondence Professor Steven S. Sharfstein, MD, Sheppard Pratt Health System, 6501 N. Charles Street, Baltimore, MD 21204, USA. Email: ssharfstein@sheppardpratt. org

\begin{abstract}
SUMMARY
Health reform in America will lead to major changes in medical care in the USA, with opportunities for improved access to care, especially for psychiatric patients. Combined with recently enacted 'parity' legislation, health reform will expand public and private insurance coverage to an additional 33 million Americans, leading to increased pressure on the psychiatrists who are willing to practise with the insured population and on opportunities for subspecialisation within the field. Some essential non-medical tasks will not be funded by insurance, and these must be given due consideration. A major emphasis of health reform is to integrate general health and mental health services. The reform will have an impact on the use of the hospital and emergency room as well. A major concern is the cost of care and how it will be controlled.

\section{DECLARATION OF INTEREST}

None.
\end{abstract}

On 23 March 2010, US President Barack Obama signed the Patient Protection and Affordable Care Act. Public Law 111-148 was modified a week later, on 30 March, when President Obama signed the Health Care and Education Affordability Reconciliation Act. The enactment of both Bills is a milestone in the history of health reform in the USA and their 2000-plus pages will fundamentally transform opportunities for care and treatment for all Americans.

The impact on psychiatric or behavioural healthcare will be profound. This legislation, in combination with the Mental Health Parity and Addiction Equity Act (in which provisions were written as a baseline requirement for the healthcare reform legislation), will change the practice of psychiatry and related mental health treatments in the USA, with far-reaching effects on patients, physicians, hospitals and mental health professionals.

\section{Key provisions}

The key provisions of the legislation include a yet to be developed benefits package that must include mental health and substance use treatment in the required essential benefits packages offered by the state-based health insurance exchanges. In addition, it requires that these benefits be offered at 'parity' with other medical and surgical benefits for all insurance plans sold within these insurance exchanges, which are created under the new law. Combined with the expansion of Medicaid, it is estimated that 32 million Americans who currently do not have insurance will now have access to mental healthcare through either private or public insurance and at parity with other medical disorders.

This reform will lead to a massive shift away from block grants and state funding towards private insurance and Medicaid to pay for treatment for mental and substance use disorders. In some estimates, the combination of the expansion of Medicaid to include people with incomes below $133 \%$ of the federal poverty level (about $\$ 14000$ for an individual) and the new insurance options will provide coverage for an additional 6 to 10 million people who will require mental health and/or substance misuse services (Albright 2010). A big question is whether there will be the capacity or supply of providers and settings to treat these individuals.

In addition to the expansion of coverage, the law has provisions to support education and research for postpartum depression, authorise grants for co-locating primary and specialty care in community-based mental health settings, develop centres of excellence for the research and treatment of depressive disorders, increase funding for community mental health centres, and develop a Medicaid emergency psychiatric demonstration project that would provide Medicaid federal reimbursement for individuals aged 21 to 65 in a so-called 'institution for mental disease' (or psychiatric hospital).

The private insurance reform under this legislation is the most sweeping change in US insurance regulation in the past 80 years. The statebased exchanges, beginning in 2014, will open each state for individuals and small employers to shop for health coverage. The high-risk pools (funding for individuals with serious medical disorders and no health insurance) established in 2010 cover 
adults with pre-existing conditions (including mental disorders), but this cover will end when the health exchanges are in place. The individual coverage mandate requires virtually all Americans to have health insurance, with significant subsidies for those on lower incomes. Beginning in 2014, companies with 50 or more employees must offer coverage to employees or pay a penalty. Insurance can have no lifetime or annual limits, cannot contain pre-existing condition exclusions or other discrimination based on health status, and must provide immediate access to insurance for people with pre-existing conditions. This reform has significant implications for individuals with mental illness since many mental disorders develop early in life. In the past, a major obstacle for individuals seeking cover for a mental disorder was a preexisting condition exclusion.

In addition, there is a state option to provide 'health homes' for policy holders with chronic conditions, and this includes coverage of individuals with persistent and serious mental illness. These homes would involve teams of health professionals and provide a comprehensive set of medical and related services to manage chronic illness.

There are many other changes in this legislation but most noteworthy is the closing of the Medicare coverage gap (the 'donut hole') - part of Medicare that requires individuals to pay $\$ 2700-\$ 6150$ out of pocket for their prescription coverage. During the next 10 years, the beneficiary co-insurance rate for this coverage gap will be narrowed down in phases.

\section{Impact on practice}

Since the early 1990s, when Hillary Clinton's effort to reform the healthcare system failed (Sharfstein 1993), the practice of psychiatry in the USA has changed considerably. Owing to managed care (Box 1) restrictions, many psychiatrists have become more biomedical in their orientation (in both the diagnostic and treatment aspects

\section{BOX 1 Managed care}

The purpose of managed care is to reduce healthcare costs by mechanisms such as economic incentives for physicians and patients to select less costly forms of care; programmes for reviewing the medical necessity of specific services; increased beneficiary cost sharing; controls on in-patient admissions and lengths of stay; selective contracting with healthcare providers; and the intensive management of high-cost healthcare cases.

(US National Library of Medicine, Medical Subject Heading (MeSh) definition: www.nlm.nih.gov/mesh/meshhome.html of care), the prescribing of psychoactive drugs has increased, and the medical management of serious mental disorders dominates psychiatric practice in both the hospital and out-patient setting. Managed care's administrative hassles have also led many psychiatrists to 'opt out' of any insurance reimbursement and insist on outof-pocket payment for care.

Further, psychiatric treatment (both the medical and non-medical aspects) has become quite specialised, with many subspecialties emerging as the science has grown. Diagnosis has become more reliable, and there are more treatment options. Psychiatric subspecialties today include general adult, child and adolescent, and geriatric psychiatry, neuropsychiatry (including traumatic brain injury, autism, stroke, dementias), psychosomatics, eating disorders, trauma, addictions, developmental disabilities and treatment-resistant psychotic disorders.

As medical tasks (Astrachan 1976) have expanded and subspecialisation has grown, one key question is whether there will be enough psychiatrists willing to treat all the additional individuals who may benefit from treatment. This is particularly true in the case of child and adolescent psychiatrists, who are very much in short supply. Practice will need to be reorganised to meet the increased demand for care. Health homes for the chronically ill and 'accountable care organisations' will grow as teams in such organisations will practise in the context of the community mental health model. Medical expertise of psychiatrists will be at a premium in these care systems, and those systems that combine hospital, day treatment and out-patient alternatives (that is, a continuum of care) will be best positioned to treat large populations of patients effectively and efficiently.

One of the major issues under health reform in relation to the medical tasks performed by psychiatrists will be how managed care utilisation review $^{\dagger}$ and cost controls will be implemented and whether the paperwork and telephonic reviews can be managed by a psychiatrist in office practice (i.e., the 'hassle factor'). Having these utilisation controls 'on parity' with those for other medical/surgical conditions is the expectation and is underscored by the recently released regulations on the parity legislation. Historically, psychiatric treatment has been under-funded and over-managed. The major cost control question will be whether too many incentives will exist for excessive reviews or paperwork, thereby discouraging psychiatrists from participating in the mainstream of healthcare reform. The other related issue is the fee structure
Managed care utilisation review is explained in Sharfstein S (2009) Letter from America: acute inpatient psychiatry bed shortages. Advances in Psychiatric Treatment 15: 402-403, Box 1. Ed. 
that will be created by the new health reform system. If the fees are too low and the hassles too high, many psychiatrists will opt out of insurance, which is often the situation today in highly managed urban environments.

\section{Essential non-medical tasks which may or may not be funded by health insurance}

After patients are stabilised symptomatically and various comorbid conditions addressed, there are a number of non-medical or reparative tasks that are necessary to rehabilitate patients and promote recovery. Whether or not these tasks are paid for by insurance has always been a challenging issue, especially in the public mental health system, which has had to fill in the gaps when their cost is not covered. They are indeed essential, especially for patients who are severely and persistently ill, in order to maintain functioning and improve opportunities for true recovery. These reparative tasks include the provision of day treatment or day care; assistance with household management skills; community outreach, including assertive community treatment teams; working with families, who are often the primary givers of care in the community; supported employment; case management needed to pull together various entitlements and assure access to needed rehabilitative services; and special education and recreational activities. There is much evidence that these tasks are cost-effective as they help to reduce hospital readmissions, prevent homelessness and promote recovery.

Today, Medicaid is the largest payer for these reparative tasks. So, the big issue is whether states will include them in their menu of covered services as Medicaid expands to a greater proportion of the population.

\section{Integration of general health and mental health services}

Increasing emphasis is given today to the provision of general medical care to people with serious mental illness, as it has been demonstrated that these individuals have excess mortality that has reached a quarter-century (25 years). This is primarily related to the lack of access to general medical care and problems related to smoking behaviour, obesity, diabetes and inattention to medical needs. Health reform that provides access to essential medical coverage for individuals who are seriously mentally ill can make a big difference. Whether these primary care services are available in community-based settings (i.e., community mental health centres, primary care settings with integrated mental health professionals, or both) is yet to be decided as we attempt to integrate these services.

The likely shape of accountable care organisations and health homes and their application to the care of people with serious mental illness are other issues that will be addressed in the coming months and years in the implementation of health reform. Will there be a role for a specialty 'mental health home'? In the training of physician psychiatrists, will the management of hypertension, diabetes and obesity be skills that the modern psychiatrist may need to add to their other medical and psychiatric skills? The impact of complex psychopharmacology on general health is certainly an area of expertise for psychiatrists. But then the treatment of these conditions may also be within the psychiatrist's purview as the 'principal physician' for patients with serious mental illness.

\section{Psychotherapy and health reform}

Psychotherapy is part of the treatment armamentarium for psychiatrists and others in the care of the mentally ill and should be reimbursed through the essential insurance benefits package because it relates to the reduction of symptoms and the promotion of function. Services that foster personal growth and development through psychotherapy should probably be reimbursed outside of the insurance system through out-ofpocket payments. The key question may be which professionals, in addition to psychiatrists and psychologists, should be eligible for insurance reimbursement for psychotherapy as the demand for these services is likely to expand or even explode with health reform. Cost control could become a major problem if more and more providers deliver psychotherapy to individuals who do not meet the criteria for a mental disorder.

\section{Involuntary treatment and social control}

One of the unique expectations of psychiatrists is related to social control. Psychiatric patients often exhibit behaviour that is unacceptable to society when they are at risk of harming others or themselves. Individuals with serious mental illness and psychosis comorbid with substance misuse, as well as individuals who present with socially deviant behaviours such as paedophilia, create scenarios for psychiatry that overlap with law enforcement. In-patient and out-patient commitment and work in public-funded forensic systems are areas of psychiatry that may or may not be reimbursed under health reforms. They may need to continue as part of a separate state-funded system of care. Regardless of the shape of health reform, treatment of individuals with mental illnesses who are in 
prison or at risk of incarceration is a priority as we are currently experiencing a national crisis of the number of seriously mentally ill people in jails and prisons.

\section{Impact of health reform on the emergency room and the hospital}

In many areas of the country, emergency rooms have been the place of first resort for individuals experiencing a psychiatric crisis. Access to beds has been reduced and many community-based services are unavailable. The role of the emergency room and the acute in-patient stay are often neglected in the thinking about health and mental health reform, but they are critical in the continuum of care. Available and appropriately supported housing is essential for many individuals to avoid mental health crises and homelessness. Emergency room gridlock due to lack of in-patient beds can be prevented with appropriate, quality out-patient services. Crisis beds are another alternative to hospital admission. But today, we lack those services in most areas of the country.

Will health reform provide the essential building blocks of a service system that will use emergency room and acute in-patient care appropriately and effectively or will we have an explosion of demand and need for these services because the out-patient network and continuum of care are frayed and inadequate? Short hospital stays are often followed by rapid relapse and readmission, so treatment services provided in hospital and community settings need to be tailored specifically to promote recovery and sustain treatment and recovery in the community.

Costs will need to be controlled as demand for care grows. It is likely that costs per care, per episode, per time period will be capped, with a special emphasis on in-patient care. Although this bundling of reimbursement must be done at parity with other medical conditions, it will lead to the expectation to treat more individuals at fewer dollars per case.

\section{Final thoughts}

There are more questions than answers about the impact of US health reform on psychiatry and people with mental illnesses. With the passage of parity legislation and the Obama health reform, there is now opportunity to redesign, reconfigure and reconceptualise the entire mental healthcare continuum. The resources may finally be available to put together the key building blocks in a more efficient and effective care system - one that moves patients away from incarceration and homelessness and into treatment and recovery.

\section{References}

Albright B (2010) Remaking behavioral healthcare. Behavioral Healthcare 30 (6): 14-8.

Astrachan BM, Levinson DJ, Adler DA (1976) The impact of national health insurance on the tasks and practice of psychiatry. Archives of General Psychiatry 33: 785-94.

Sharfstein SS, Stoline AM, Goldman HH (1993) Psychiatric care and health insurance reform. American Journal of Psychiatry 150: 7-18. 